International Journal of Pure and Applied Mathematics

Volume 112 No. 4 2017, 795-804

ISSN: 1311-8080 (printed version); ISSN: 1314-3395 (on-line version)

url: http://www.ijpam.eu

doi: 10.12732/ijpam.v112i4.10

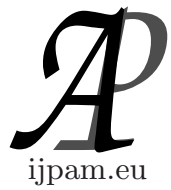

\title{
APPROXIMATION BY EXPONENTIAL TYPE VECTORS OF POSITIVE OPERATORS
}

\author{
Marian Dmytryshyn \\ Vasyl Stefanyk Precarpathian National University \\ 57 Shevchenka str., 76018, Ivano-Frankivsk, UKRAINE
}

\begin{abstract}
We establish the estimations of best approximations of elements of a Banach space by exponential type vectors associated with the positive operator. The corresponding estimations are expressed in terms of quasi-norms of the approximation spaces as BernsteinJackson-type inequalities. Such inequalities are applied to spectral approximations in the case of the positive operator with the point spectrum.
\end{abstract}

AMS Subject Classification: $47 \mathrm{~A} 58,41 \mathrm{~A} 17$

Key Words: exponential type entire vectors, Bernstein-Jackson-type inequalities, spectral approximations

\section{Introduction and preliminaries}

One of the problems in the approximation theory is to characterize the approximation of elements of Banach space by different classes of smooth vectors associated with a closed unbounded operator. A characterization of some classes of infinitely differentiable vectors of a normal operator in a Hilbert space is given in terms of the rate with which the best approximation of the vectors by entire exponential type vectors tend to zero [1]. The spaces of exponential type entire vectors associated with the closed unbounded linear operator in a Banach space are defined in [2]. The direct and inverse theorems in the theory of approximations are established in [3] and [4].

$\begin{array}{ll}\text { Received: } & \text { February 1, 2017 } \\ \text { Revised: } & \text { February 14, 2017 } \\ \text { Published: } & \text { February 19, 2017 }\end{array}$

(c) 2017 Academic Publications, Ltd. url: www.acadpubl.eu 
The motivation of our work is to investigate a best approximation problem by invariant subspaces of exponential type vectors of a positive operator in a Banach space. Such problem for any closed unbounded linear operator has been studied in [5] and [6]. In this paper, we define the Lorentz-type interpolation spaces of exponential type vectors of positive operators and appropriate approximation spaces. Interpolation properties of such spaces are explored (Theorems 1, 2).

The inequalities estimating the minimal distance from a given element to a subspace of exponential type vectors are established (Theorem 4). Such inequalities are extended to cases of best spectral approximations of the positive operator in a Banach space (Theorem 5).

In a Banach complex space $(\mathbf{X},\|\cdot\|)$ we consider a positive operator $A$ with the norm dense domain $\mathrm{D}^{1}(A)$. It means that $(-\infty, 0] \in \rho(A)$ and there exists a number $c>0$ such that $\left\|(A-\lambda I)^{-1}\right\| \leq c /(1+|\lambda|)$ for all $\lambda \in(-\infty, 0]$ ([7], Section 1.14.1). Let $\mathrm{D}^{k+1}(A)=\left\{x \in \mathrm{D}^{k}(A): A^{k} x \in \mathrm{D}(A)\right\}$ and $\mathrm{D}^{\infty}(A)=$ $\bigcap_{k \in \mathbb{N}} \mathrm{D}^{k}(A)$. We accept that $A^{0}$ is the unit operator on $\mathbf{X}$.

Recall the real ( $K$-functional) and complex interpolation methods (for more details see [7] and [8]). Let $0<\theta<1$ and $1 \leq q \leq \infty$. For the quasinormed spaces $X_{0}$ and $X_{1}$ we define the interpolation space $\left(X_{0}, X_{1}\right)_{\theta, q}=\{x \in$ $\left.X_{0}+X_{1}:|x|_{\left(X_{0}, X_{1}\right)_{\theta, q}}<\infty\right\}$ with the quasi-norm

$$
|x|_{\left(X_{0}, X_{1}\right)_{\theta, q}}= \begin{cases}\left(\int_{0}^{\infty}\left[t^{-\theta} K\left(t, x ; X_{0}, X_{1}\right)\right]^{q} \frac{d t}{t}\right)^{1 / q}, & 1 \leq q<\infty, \\ \sup _{0<t<\infty} t^{-\theta} K\left(t, x ; X_{0}, X_{1}\right), & q=\infty .\end{cases}
$$

where $K\left(t, x ; X_{0}, X_{1}\right)=\inf _{x=x^{0}+x^{1}}\left(\left|x^{0}\right|_{X_{0}}+t\left|x^{1}\right|_{X_{1}}\right), t>0$.

For any Banach spaces $X_{0}$ and $X_{1}$ we define the interpolation space $\left[X_{0}, X_{1}\right]_{\theta}$ $=\left\{x \in X_{0}+X_{1}: \exists f(z) \in \mathcal{F}\left(X_{0}, X_{1}\right), f(\theta)=x\right\}$ with the norm $\|x\|_{\left[X_{0}, X_{1}\right]_{\theta}}=$ $\inf _{f(\theta)=x}\|f(z)\|_{\mathcal{F}\left(X_{0}, X_{1}\right)}$, where inf takes on all functions $f \in \mathcal{F}\left(X_{0}, X_{1}\right)$ such that $f(\theta)=x$. By $\mathcal{F}\left(X_{0}, X_{1}\right)$ we denote the space of $\left(X_{0}+X_{1}\right)$-valued functions with the norm $\|f\|_{\mathcal{F}\left(X_{0}, X_{1}\right)}=\max \left(\sup _{t}\|f(i t)\|_{X_{0}}, \sup _{t}\|f(1+i t)\|_{X_{1}}\right), f(i t) \in X_{0}$, $f(1+i t) \in X_{1}$ for all $-\infty<t<\infty$.

\section{Lorentz-Type Spaces}

Let $m, k \in \mathbb{Z}, m \geq 0$. It is well known ([7], Section 1.15.1) that for all $\alpha \in \mathbb{C}$ such that $-m<\operatorname{Re} \alpha \leq \sigma-m, 0<\sigma<k$ and all $x \in\left(\mathbf{X}, \mathrm{D}^{k}(A)\right)_{\sigma / k, 1}$ is defined 
an operator

$$
A_{\sigma}^{\alpha} x=\frac{\Gamma(k)}{\Gamma(\alpha+m) \Gamma(k-m-\alpha)} \int_{0}^{\infty} t^{\alpha+m-1} A^{k-m}(A+t I)^{-k} x d t .
$$

The operator $A_{\sigma}^{\alpha}$ has a closure $A^{\alpha}$ in $\mathrm{X}$ which is independent of $\sigma$. The domain $\mathrm{D}^{\alpha}:=\mathrm{D}^{\alpha}(A)$ of $A^{\alpha}$ we consider as a Banach space with the norm $\|x\|_{\mathrm{D}^{\alpha}}=$ $\left\|A^{\alpha} x\right\|, x \in \mathrm{D}^{\alpha}$.

Let $x_{k, \nu}:=(A / \nu)^{k} x(\nu>0)$ and a sequence $\left(x_{k, \nu}^{*}\right)_{k=0}^{\infty}$ consists of elements $x_{k, \nu}$ which are numbered in according to decrease of norms

$$
\left\|x_{0, \nu}^{*}\right\|_{\mathrm{D}^{\alpha}} \geq\left\|x_{1, \nu}^{*}\right\|_{\mathrm{D}^{\alpha}} \geq \ldots \geq\left\|x_{k, \nu}^{*}\right\|_{\mathrm{D}^{\alpha}} \geq \ldots
$$

For any $0<\nu<\infty$ and $1 \leq q, p \leq \infty$ we define the space $\mathcal{E}_{q, p}^{\nu, \alpha}(A)=$ $\left\{x \in \mathrm{D}^{\infty}(A):\|x\|_{\mathcal{E}_{q, p}^{\nu, \alpha}(A)}<\infty\right\}$, where

$$
\|x\|_{\mathcal{E}_{q, p}^{\nu, \alpha}(A)}= \begin{cases}\left(\sum_{k \in \mathbb{N}}\left\|x_{k-1, \nu}^{*}\right\|_{\mathrm{D}^{\alpha}}^{p} k^{\frac{p}{q}-1}\right)^{1 / p}, & 1 \leq p<\infty \\ \sup _{k \in \mathbb{N}}\left\|x_{k-1, \nu}^{*}\right\|_{\mathrm{D}^{\alpha} k^{\frac{1}{q}},} & p=\infty .\end{cases}
$$

We can call the space $\mathcal{E}_{q, p}^{\nu, \alpha}(A)$ endowed with the norm $\|x\|_{\mathcal{E}_{q, p}^{\nu, \alpha}(A)}$ a Lorentztype space of exponential type entire vectors of $A$. If $q=p$ then $\mathcal{E}_{q, q}^{\nu, \alpha}(A)=$ $\mathcal{E}_{q}^{\nu, \alpha}(A)$ and $\mathcal{E}_{1,1}^{\nu, \alpha}(A)=\mathcal{E}_{1}^{\nu, \alpha}(A), \mathcal{E}_{\infty, \infty}^{\nu, \alpha}(A)=\mathcal{E}_{\infty}^{\nu, \alpha}(A)$.

Applying [6, Theorem 2.1] for $1<q<\infty$ and $1 \leq p \leq \infty$, we obtain

$$
\left(\mathcal{E}_{1}^{\nu, \alpha}(A), \mathcal{E}_{\infty}^{\nu, \alpha}(A)\right)_{1-1 / q, p}=\mathcal{E}_{q, p}^{\nu, \alpha}(A)
$$

with equivalent norms. Moreover, the embedding $\mathcal{E}_{q, p}^{\nu, \alpha}(A) \subset \mathcal{E}_{q, p}^{\mu, \alpha}(A)$ with $\mu>\nu$ holds and every space $\mathcal{E}_{q, p}^{\nu, \alpha}(A)$ is complete.

Theorem 1. Let $0<\nu<\infty, \alpha \in \mathbb{C}, 1<q_{0}, q_{1}<\infty, q_{0} \neq q_{1}, 1 \leq$ $p, p_{0}, p_{1} \leq \infty$ and $0<\theta<1$. Then the following equality holds:

$$
\left(\mathcal{E}_{q_{0}, p_{0}}^{\nu, \alpha}(A), \mathcal{E}_{q_{1}, p_{1}}^{\nu, \alpha}(A)\right)_{\theta, p}=\mathcal{E}_{q, p}^{\nu, \alpha}(A), \frac{1}{q}=\frac{1-\theta}{q_{0}}+\frac{\theta}{q_{1}} .
$$

Proof. By Theorem 3.11.5 [8] and (1) we have

$$
\left(\mathcal{E}_{q_{0}, p_{0}}^{\nu, \alpha}(A), \mathcal{E}_{q_{1}, p_{1}}^{\nu, \alpha}(A)\right)_{\theta, p}=\left(\mathcal{E}_{1}^{\nu, \alpha}(A), \mathcal{E}_{\infty}^{\nu, \alpha}(A)\right)_{\lambda, p}=\mathcal{E}_{1 /(1-\lambda), p}^{\nu, \alpha}(A)
$$

where $q_{0}=1 /\left(1-\theta_{0}\right), q_{1}=1 /\left(1-\theta_{1}\right), \lambda=(1-\theta) \theta_{0}+\theta \theta_{1}, 0 \leq \theta_{0}<\theta_{1} \leq 1$. Putting $q=1 /(1-\lambda)$ in (3), we obtain (2). 
For any $1 \leq q \leq \infty, \alpha, \beta \in \mathbb{C}$ and $0 \leq \operatorname{Re} \alpha<\operatorname{Re} \beta<\infty$ we define the spaces $\mathcal{E}_{q, \theta}^{\nu,(\alpha, \beta)}(A)=\left\{x \in \mathrm{D}^{\infty}(A):\|x\|_{\mathcal{E}_{q, \theta}^{\nu,(\alpha, \beta)}(A)}<\infty\right\}$,

$$
\|x\|_{\mathcal{E}_{q, \theta}^{\nu,(\alpha, \beta)}(A)}= \begin{cases}\left(\sum_{k \in \mathbb{Z}_{+}}\left\|x_{k, \nu}\right\|_{\left(\mathrm{D}^{\alpha}, \mathrm{D}^{\beta}\right)_{\theta, q}}^{q}\right)^{1 / q}, & 1 \leq q<\infty, \\ \sup _{k \in \mathbb{Z}_{+}}\left\|x_{k, \nu}\right\|_{\left(\mathrm{D}^{\alpha}, \mathrm{D}^{\beta}\right)_{\theta, q}}, & q=\infty .\end{cases}
$$

and $\mathcal{E}_{q, \theta}^{\nu,[\alpha, \beta]}(A)=\left\{x \in \mathrm{D}^{\infty}(A):\|x\|_{\mathcal{E}_{q, \theta}^{\nu,[\alpha, \beta]}(A)}<\infty\right\}$,

$$
\|x\|_{\mathcal{E}_{q, \theta}^{\nu,[\alpha, \beta]}(A)}= \begin{cases}\left(\sum_{k \in \mathbb{Z}_{+}}\left\|x_{k, \nu}\right\|_{\left[\mathrm{D}^{\alpha}, \mathrm{D}^{\beta}\right]_{\theta}}^{q}\right)^{1 / q}, & 1 \leq q<\infty, \\ \sup _{k \in \mathbb{Z}_{+}}\left\|x_{k, \nu}\right\|_{\left[\mathrm{D}^{\alpha}, \mathrm{D}^{\beta}\right]_{\theta}}, & q=\infty .\end{cases}
$$

Theorem 2. Let $0<\theta<1, \alpha, \beta \in \mathbb{C}, 0 \leq \operatorname{Re} \alpha<\operatorname{Re} \beta<\infty$ and $1 \leq q, q_{0}, q_{1} \leq \infty$. If $0<\nu_{0}, \nu_{1}<\infty, \nu_{0} \neq \nu_{1}$ then for $\nu=\nu_{0}^{1-\theta} \nu_{1}^{\theta}$ the following equality holds:

$$
\left(\mathcal{E}_{q_{0}}^{\nu_{0}, \alpha}(A), \mathcal{E}_{q_{1}}^{\nu_{1}, \alpha}(A)\right)_{\theta, q}=\mathcal{E}_{q}^{\nu, \alpha}(A)
$$

For $1 \leq q_{0}, q_{1}<\infty$ such that $1 / q=(1-\theta) / q_{0}+\theta / q_{1}$ the following equalities hold:

$$
\begin{gathered}
\left(\mathcal{E}_{q_{0}}^{\nu, \alpha}(A), \mathcal{E}_{q_{1}}^{\nu, \beta}(A)\right)_{\theta, q}=\mathcal{E}_{q, \theta}^{\nu,(\alpha, \beta)}(A) . \\
{\left[\mathcal{E}_{q_{0}}^{\nu_{0}, \alpha}(A), \mathcal{E}_{q_{1}}^{\nu_{1}, \alpha}(A)\right]_{\theta}=\mathcal{E}_{q}^{\nu, \alpha}(A),} \\
{\left[\mathcal{E}_{q_{0}}^{\nu, \alpha}(A), \mathcal{E}_{q_{1}}^{\nu, \beta}(A)\right]_{\theta}=\mathcal{E}_{q, \theta}^{\nu,[\alpha, \beta]}(A) .}
\end{gathered}
$$

Proof. The space $\mathcal{E}_{q}^{\nu, \alpha}(A)$ is isometric to the space of sequences $l_{q}^{\nu, \alpha}=\{\bar{x}:=$ $\left.\left(A^{k} x\right)_{k=0}^{\infty}: x \in \mathcal{E}_{q}^{\nu, \alpha}(A)\right\}$ with the norm $\|\bar{x}\|_{l_{q}^{\nu, \alpha}}=\|x\|_{\mathcal{E}_{q}^{\nu, \alpha}(A)}$. Let us replace $\nu=2^{-\sigma}$ in which the condition $\nu=\nu_{0}^{1-\theta} \nu_{1}^{\theta}$ turns into equality $\sigma=(1-\theta) \sigma_{0}+$ $\theta \sigma_{1}$. Moreover, we have $K\left(t, \bar{x}, l_{\infty}^{\nu_{0}, \alpha}, l_{\infty}^{\nu_{1}, \alpha}\right) \sim \sup _{k \in \mathbb{Z}_{+}} \min \left(2^{k \sigma_{0}}, t 2^{k \sigma_{1}}\right)\left\|A^{k} x\right\|_{\mathrm{D}^{\alpha}}$ for $q_{0}=q_{1}=\infty$ and $K\left(t, \bar{x}, l_{1}^{\nu_{0}, \alpha}, l_{1}^{\nu_{1}, \alpha}\right) \sim \sum_{k \in \mathbb{N}} \min \left(2^{k \sigma_{0}}, t 2^{k \sigma_{1}}\right)\left\|A^{k} x\right\|_{\mathrm{D}^{\alpha}}$ for $q_{0}=q_{1}=1$.

For $\bar{x} \in\left(l_{\infty}^{\nu_{0}, \alpha}, l_{\infty}^{\nu_{1}, \alpha}\right)_{\theta, q}$ and $\sigma_{0}>\sigma_{1}$ we obtain

$$
\|\bar{x}\|_{\left(l_{\infty}^{\nu_{0}, \alpha}, l_{\infty}^{\nu_{1}, \alpha}\right)_{\theta, q}}^{q}=\int_{0}^{\infty}\left[t^{-\theta} K\left(t, \bar{x} ; l_{\infty}^{\nu_{0}, \alpha}, l_{\infty}^{\nu_{1}, \alpha}\right)\right]^{q} \frac{d t}{t}
$$




$$
\begin{aligned}
& \sim \sum_{j \in \mathbb{Z}} 2^{-\theta q j\left(\sigma_{0}-\sigma_{1}\right)} \sup _{k}\left[\min \left(2^{k \sigma_{0}}, 2^{j\left(\sigma_{0}-\sigma_{1}\right)+k \sigma_{1}}\right)\left\|A^{k} x\right\|_{\mathrm{D}^{\alpha}}\right]^{q} \\
& \geq \sum_{j \in \mathbb{Z}} 2^{q j\left[\sigma_{0}(1-\theta)+\sigma_{1} \theta\right]}\left\|A^{j} x\right\|_{\mathrm{D}^{\alpha}}^{q}=c\|\bar{x}\|_{l_{q}^{\nu, \alpha}}^{q}
\end{aligned}
$$

which shows the embedding

$$
\left(l_{\infty}^{\nu_{0}, \alpha}, l_{\infty}^{\nu_{1}, \alpha}\right)_{\theta, q} \subset l_{q}^{\nu, \alpha}
$$

Using the Hölder's inequality, for $\bar{x} \in l_{q}^{\nu, \alpha}$ we have

$$
\begin{aligned}
& \|\bar{x}\|_{\left(l_{1}^{\nu_{0}, \alpha}, l_{1}^{\nu_{1}, \alpha}\right)_{\theta, q}}^{q}=\int_{0}^{\infty}\left[t^{-\theta} K\left(t, \bar{x} ; l_{1}^{\nu_{0}, \alpha}, l_{1}^{\nu_{1}, \alpha}\right)\right]^{q} \frac{d t}{t} \\
& \sim \sum_{j \in \mathbb{Z}} 2^{-\theta q j\left(\sigma_{0}-\sigma_{1}\right)}\left[\sum_{k \in \mathbb{Z}_{+}} \min \left(2^{k \sigma_{0}}, 2^{j\left(\sigma_{0}-\sigma_{1}\right)+k \sigma_{1}}\right)\left\|A^{k} x\right\|_{\mathrm{D}^{\alpha}}\right]^{q} \\
& \leq c^{\prime} \sum_{j \in \mathbb{Z}} 2^{q j\left(\sigma-\sigma_{0}\right)}\left(\sum_{k \leq j} 2^{q^{\prime} k\left(\sigma_{0}-\mu_{0}\right)}\right)^{q / q^{\prime}}\left(\sum_{k \leq j} 2^{q k \mu_{0}}\left\|A^{k} x\right\|_{\mathrm{D}^{\alpha}}^{q}\right) \\
& \quad+c^{\prime} \sum_{j \in \mathbb{Z}} 2^{q j\left(\sigma-\sigma_{1}\right)}\left(\sum_{k>j} 2^{q^{\prime} k\left(\sigma_{1}-\mu_{1}\right)}\right)^{q / q^{\prime}}\left(\sum_{k>j} 2^{q k \mu_{1}}\left\|A^{k} x\right\|_{\mathrm{D}^{\alpha}}^{q}\right) \\
& \leq c^{\prime \prime}\left(\sum_{k \in \mathbb{Z}} 2^{q k \mu_{0}}\left\|A^{k} x\right\|_{\mathrm{D}^{\alpha}}^{q} \sum_{j \geq k} 2^{q j\left(\sigma-\mu_{0}\right)}\right. \\
& \left.+\sum_{k \in \mathbb{Z}} 2^{q k \mu_{1}}\left\|A^{k} x\right\|_{\mathrm{D}^{\alpha}}^{q} \sum_{j<k} 2^{q j\left(\sigma-\mu_{1}\right)}\right) \leq c \sum_{k \in \mathbb{Z}} 2^{k \sigma q}\left\|A^{k} x\right\|_{\mathrm{D}^{\alpha}}^{q}=c\left\|_{\bar{x}}\right\|_{l_{q}^{\nu, \alpha} .}^{q}
\end{aligned}
$$

It follows that

$$
l_{q}^{\nu, \alpha} \subset\left(l_{1}^{\nu_{0}, \alpha}, l_{1}^{\nu_{1}, \alpha}\right)_{\theta, q} .
$$

From (8) and (9) we obtain

$$
l_{q}^{\nu, \alpha} \subset\left(l_{1}^{\nu_{0}, \alpha}, l_{1}^{\nu_{1}, \alpha}\right)_{\theta, q} \subset\left(l_{q_{0}}^{\nu_{0}, \alpha}, l_{q_{1}}^{\nu_{1}, \alpha}\right)_{\theta, q} \subset\left(l_{\infty}^{\nu_{0}, \alpha}, l_{\infty}^{\nu_{1}, \alpha}\right)_{\theta, q} \subset l_{q}^{\nu, \alpha} .
$$

Thus we have the required equality (4).

The space $\mathcal{E}_{q}^{\nu, \alpha}(A)$ is isometric embedding in the space $l_{q}^{\alpha}=\left\{\left(\xi_{k}\right)_{k=0}^{\infty}: \xi_{k} \in\right.$ $\left.\mathrm{D}^{\alpha},\left\|\left(\xi_{k}\right)\right\|_{l_{q}^{\alpha}}=\left(\sum_{k \in \mathbb{Z}_{+}}\left\|\xi_{k}\right\|_{\mathrm{D}^{\alpha}}^{q}\right)^{1 / q}<\infty\right\}$. Denote $\mathcal{I}: \mathcal{E}_{q}^{\nu, \alpha}(A) \ni x \hookrightarrow\left(\xi_{k}=\right.$ $\left.x_{k, \nu}\right) \in l_{q}^{\alpha}$. For $x \in\left(l_{q_{0}}^{\alpha}, l_{q_{1}}^{\beta}\right)_{\theta, q}$ we have

$$
\begin{aligned}
& K\left(t, \mathcal{I}(x), l_{q_{0}}^{\alpha}, l_{q_{1}}^{\beta}\right) \leq \inf _{x=x_{0}+x_{1}}\left(\left\|\mathcal{I}\left(x_{0}\right)\right\|_{l_{q_{0}}^{\alpha}}+t\left\|\mathcal{I}\left(x_{1}\right)\right\|_{l_{q_{1}}^{\beta}}\right) \\
& \quad \leq\|\mathcal{I}\|_{\mathcal{E}_{q_{0}}^{\nu, \alpha}(A) \rightarrow l_{q_{0}}^{\alpha}} K\left(\frac{t\|\mathcal{I}\|_{\mathcal{E}_{q_{1}}^{\nu, \beta}(A) \rightarrow l_{q_{1}}^{\beta}}}{\|\mathcal{I}\|_{\mathcal{E}_{q_{0}}^{\nu, \alpha}(A) \rightarrow l_{q_{0}}^{\alpha}}}, x, \mathcal{E}_{q_{0}}^{\nu, \alpha}(A), \mathcal{E}_{q_{1}}^{\nu, \beta}(A)\right) .
\end{aligned}
$$


Through a substitution $\tau=t\|\mathcal{I}\|_{\mathcal{E}_{q_{1}}^{\nu, \beta}(A) \rightarrow l_{q_{1}}^{\beta}}\|\mathcal{I}\|_{\mathcal{E}_{q_{0}}^{\nu, \alpha}(A) \rightarrow l_{q_{0}}^{\alpha}}^{-1}$ we obtain

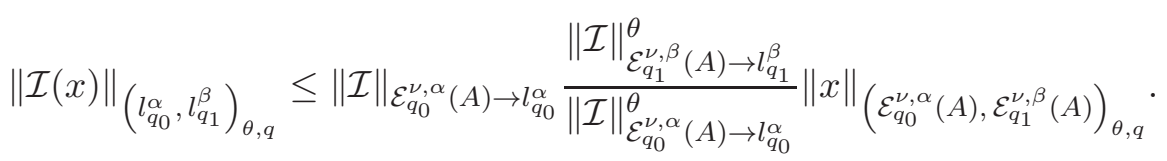

Applying the similar estimate for the inverse mapping $\mathcal{I}^{-1}$ defined on the set of values of $\mathcal{I}$, we obtain

$$
\|\mathcal{I}(x)\|_{\left(l_{q_{0}}^{\alpha}, l_{q_{1}}^{\beta}\right)_{\theta, q}}=\|x\|_{\left(\mathcal{E}_{q_{0}}^{\nu, \alpha}(A), \mathcal{E}_{q_{1}}^{\nu, \beta}(A)\right)_{\theta, q}}, x \in\left(\mathcal{E}_{q_{0}}^{\nu, \alpha}(A), \mathcal{E}_{q_{1}}^{\nu, \beta}(A)\right)_{\theta, q} .
$$

Every space $\mathcal{E}_{q, \theta}^{\nu,(\alpha, \beta)}(A)$ is isometric to the space of sequences $l_{q, \theta}^{\nu,(\alpha, \beta)}=\{\bar{x}:=$ $\left.\left(A^{k} x\right)_{k=0}^{\infty}: x \in \mathcal{E}_{q, \theta}^{\nu,(\alpha, \beta)}(A)\right\}$ endowed with the norm $\|\bar{x}\|_{l_{q, \theta}^{\nu,(\alpha, \beta)}}=\|x\|_{\mathcal{E}_{q, \theta}^{\nu,(\alpha, \beta)}(A)}$. Applying [7, Theorem 1.18.1], we obtain $\left(l_{q_{0}}^{\alpha}, l_{q_{1}}^{\beta}\right)_{\theta, q}=l_{q, \theta}^{(\alpha, \beta)}$, where $l_{q, \theta}^{(\alpha, \beta)}=$ $\left\{\left(\xi_{k}\right)_{k=0}^{\infty}: \xi_{k} \in\left(\mathrm{D}^{\alpha}, \mathrm{D}^{\beta}\right)_{\theta, q},\left\|\left(\xi_{k}\right)\right\|_{l_{q, \theta}^{(\alpha, \beta)}}^{q}=\sum_{k \in \mathbb{Z}_{+}}\left\|\xi_{k}\right\|_{\left(\mathrm{D}^{\alpha}, \mathrm{D}^{\beta}\right)_{\theta, q}}^{q}<\infty\right\}$ and $1 / q=(1-\theta) / q_{0}+\theta / q_{1}$. It follows that (5) is true.

The equality (6) follows immediately from (4) and Theorem 4.7 .2 [8].

Let $x \in\left[\mathcal{E}_{q_{0}}^{\nu, \alpha}(A), \mathcal{E}_{q_{1}}^{\nu, \beta}(A)\right]_{\theta}, f(z) \in \mathcal{F}\left(\mathcal{E}_{q_{0}}^{\nu, \alpha}(A), \mathcal{E}_{q_{1}}^{\nu, \beta}(A)\right)$ and $f(\theta)=x$. Then

$$
g(z)=\left(\frac{\|\mathcal{I}\|_{\mathcal{E}_{q_{0}}^{\nu, \alpha}(A) \rightarrow l_{q_{0}}^{\alpha}}}{\|\mathcal{I}\|_{\mathcal{E}_{q_{1}}^{\nu, \beta}(A) \rightarrow l_{q_{1}}^{\beta}}^{\beta}}\right)^{z-\theta} \mathcal{I} f(z) \in \mathcal{F}\left(l_{q_{0}}^{\alpha}, l_{q_{1}}^{\beta}\right), g(\theta)=\mathcal{I} x .
$$

It follows that

$$
\begin{aligned}
\|g(z)\|_{\mathcal{F}\left(l_{q_{0}}^{\alpha}, l_{q_{1}}^{\beta}\right)} & \leq\|\mathcal{I}\|_{\mathcal{E}_{q_{0}}^{\nu, \alpha}(A) \rightarrow l_{q_{0}}^{\alpha}}^{(1-\theta)}\|\mathcal{I}\|_{\mathcal{E}_{q_{1}}^{\nu, \beta}(A) \rightarrow l_{q_{1}}^{\beta}}^{\theta}\|f(z)\|_{\mathcal{F}\left(\mathcal{E}_{q_{0}}^{\nu, \alpha}(A), \mathcal{E}_{q_{1}}^{\nu, \beta}(A)\right)}, \\
\|\mathcal{I} x\|_{\left[l_{q_{0}}^{\alpha}, l_{q_{1}}^{\beta}\right]_{\theta}} & \leq\|\mathcal{I}\|_{\mathcal{E}_{q_{0}}^{\nu, \alpha}(A) \rightarrow l_{q_{0}}^{\alpha}}^{(1-\theta)}\|\mathcal{I}\|_{\mathcal{E}_{q_{1}}^{\nu, \beta}(A) \rightarrow l_{q_{1}}^{\beta}}^{\theta}\|x\|_{\left[\mathcal{E}_{q_{0}}^{\nu, \alpha}(A), \mathcal{E}_{q_{1}}^{\nu, \beta}(A)\right]_{\theta}} .
\end{aligned}
$$

Applying the similar estimate for the inverse mapping $\mathcal{I}^{-1}$, we obtain

$$
\|\mathcal{I}(x)\|_{\left[l_{q_{0}}^{\alpha}, l_{q_{1}}^{\beta}\right]_{\theta}}=\|x\|_{\left[\mathcal{E}_{q_{0}}^{\nu, \alpha}(A), \mathcal{E}_{q_{1}}^{\nu, \beta}(A)\right]_{\theta}} \text { for all } x \in\left[\mathcal{E}_{q_{0}}^{\nu, \alpha}(A), \mathcal{E}_{q_{1}}^{\nu, \beta}(A)\right]_{\theta} .
$$

The space $\mathcal{E}_{q, \theta}^{\nu,[\alpha, \beta]}(A)$ is isometric to the space of sequences $l_{q, \theta}^{\nu,[\alpha, \beta]}=\{\bar{x}:=$ $\left.\left(A^{k} x\right)_{k=0}^{\infty}: x \in \mathcal{E}_{q, \theta}^{\nu,[\alpha, \beta]}(A)\right\}$ with the norm $\|\bar{x}\|_{l_{q, \theta}^{\nu,[\alpha, \beta]}}=\|x\|_{\mathcal{E}_{q, \theta}^{\nu,[\alpha, \beta]}(A)}$.

By Theorem 1.18.1 [7] we have $\left[l_{q_{0}}^{\alpha}, l_{q_{1}}^{\beta}\right]_{\theta}=l_{q, \theta}^{[\alpha, \beta]}$, where $l_{q, \theta}^{[\alpha, \beta]}=\left\{\left(\xi_{k}\right)_{k=0}^{\infty}\right.$ : $\left.\xi_{k} \in\left[\mathrm{D}^{\alpha}, \mathrm{D}^{\beta}\right]_{\theta},\left\|\left(\xi_{k}\right)\right\|_{l_{q, \theta}^{[\alpha, \beta]}}^{q}=\sum_{k \in \mathbb{Z}_{+}}\left\|\xi_{k}\right\|_{\left[\mathrm{D}^{\alpha}, \mathrm{D}^{\beta}\right]_{\theta}}^{q}<\infty\right\}$ and $1 / q=(1-\theta) / q_{0}+$ $\theta / q_{1}$. It follows that $(7)$ is true. 


\section{Best Approximations}

On the linear manifold $\mathcal{E}_{q, p}^{\alpha}(A)=\bigcup_{\nu>0} \mathcal{E}_{q, p}^{\nu, \alpha}(A)$ we define the quasi-norm $|x|_{\mathcal{E}_{q, p}^{\alpha}(A)}$ $=\|x\|+\inf \left\{\nu>0: x \in \mathcal{E}_{q, p}^{\nu, \alpha}(A)\right\}$. Given a pair of numbers $\{0<s<\infty, 0<$ $\tau \leq \infty\}$ or $\{0 \leq s<\infty, \tau=\infty\}$ we consider the scale of approximation spaces $\mathcal{B}_{q, p, \tau}^{s, \alpha}(A)=\left\{x \in \mathbf{X}:|x|_{\mathcal{B}_{q, p, \tau}^{s, \alpha}(A)}<\infty\right\}$

$$
|x|_{\mathcal{B}_{q, p, \tau}^{s, \alpha}(A)}= \begin{cases}\left(\int_{0}^{\infty}\left[t^{\alpha} E_{q, p}^{\alpha}(t, x)\right]^{\tau} \frac{d t}{t}\right)^{1 / \tau}, & 0<\tau<\infty \\ \sup _{t>0} t^{\alpha} E_{q, p}^{\alpha}(t, x), & \tau=\infty\end{cases}
$$

where $E_{q, p}^{\alpha}(t, x)=\inf \left\{\left\|x-x^{0}\right\|: x^{0} \in \mathcal{E}_{q, p}^{\alpha}(A),\left|x^{0}\right|_{\mathcal{E}_{q, p}^{\alpha}(A)} \leq t\right\}, x \in \mathbf{X}$.

Let $\left[\mathcal{B}_{q, p, \tau}^{s, \alpha}(A)\right]^{\theta}$ be the space $\mathcal{B}_{q, p, \tau}^{s, \alpha}(A)$ endowed with the quasi-norm $|x|_{\mathcal{B}_{q, p, \tau}^{s, \alpha}(A)}^{\theta}$, $x \in \mathcal{B}_{q, p, \tau}^{s, \alpha}(A)$. Applying Theorem 7.1.7 [8], we obtain

$$
\left[\mathcal{B}_{q, p, \tau}^{s, \alpha}(A)\right]^{\theta}=\left(\mathcal{E}_{q, p}^{\alpha}(A), \mathbf{X}\right)_{\theta, g}, \quad \theta=1 /(s+1), \tau=g \theta,
$$

with equivalent quasi-norms.

Lemma 3. Every space $\mathcal{B}_{q, p, \tau}^{s, \alpha}(A)$ is complete.

Proof. From the inequality $\|x\| \leq|x|_{\mathcal{E}_{q, p}^{\alpha}(A)}, x \in \mathcal{E}_{q, p}^{\alpha}(A)$ it follows that $\|x\|=\inf _{x=x^{0}+x^{1}}\left(\left|x^{0}\right|_{\mathcal{E}_{q, p}^{\alpha}(A)}+\left\|x^{1}\right\|\right)$ with $x^{0} \in \mathcal{E}_{q, p}^{\alpha}(A)$ and $x^{1} \in \mathrm{X}$. Thus, every series $\sum_{n \in \mathbb{N}} x_{n}$ with $x_{n} \in\left(\mathcal{E}_{q, p}^{\alpha}(A), \mathrm{X}\right)_{\theta, g}$ such that $\sum_{n \in \mathbb{N}}\left|x_{n}\right|_{\left(\mathcal{E}_{q, p}^{\alpha}(A), \mathrm{X}\right)_{\theta, g}}<$ $\infty$ is convergent to $x \in \mathrm{X}$. Using the inequality $\left|\sum_{n \in \mathbb{N}} x_{n}\right|_{\left(\mathcal{E}_{q, p}^{\alpha}(A), \mathrm{X}\right)_{\theta, g}} \leq$ $\sum_{n \in \mathbb{N}}\left|x_{n}\right|_{\left(\mathcal{E}_{q, p}^{\alpha}(A), \mathrm{X}\right)_{\theta, g}}$ we obtain $x \in\left(\mathcal{E}_{q, p}^{\alpha}(A), \mathrm{X}\right)_{\theta, g}$. So, $\left(\mathcal{E}_{q, p}^{\alpha}(A), \mathrm{X}\right)_{\theta, g}$ is complete. The isomorphism (10) implies that the space $\left.\mathcal{B}_{q, p, \tau}^{s, \alpha}(A)\right)$ is complete.

Theorem 4. There are constants $c_{1}$ and $c_{2}$ such that the following inequalities hold:

$$
\begin{aligned}
|x|_{\mathcal{B}_{q, p, \tau}^{s, \alpha}(A)} & \leq c_{1}|x|_{\mathcal{E}_{q, p}^{\alpha}(A)}^{s}\|x\|, \quad x \in \mathcal{E}_{q, p}^{\alpha}(A), \\
E_{q, p}^{\alpha}(t, x) & \leq c_{2} t^{-s}|x|_{\mathcal{B}_{q, p, \tau}^{s, \alpha}(A)}, \quad x \in \mathcal{B}_{q, p, \tau}^{s, \alpha}(A) .
\end{aligned}
$$

Proof. Applying [8, Theorem 3.11.4(b)], for some constant $c(\theta, g)$ we obtain

$$
|x|_{\left(\mathcal{E}_{q, p}^{\alpha}(A), \mathrm{X}\right)_{\theta, g}} \leq c|x|_{\mathcal{E}_{q, p}^{\alpha}(A)}^{1-\theta}\|x\|^{\theta}, \quad x \in \mathcal{E}_{q, p}^{\alpha}(A) .
$$

This inequality and the isomorphism (10) imply that there is a constant $c_{1}(s, \tau)$ such that the inequality (11) is true. 
By [8, Theorem 3.11.4(a)] for some constant $c(\theta, g)$ we have

$$
K\left(t, x ; \mathcal{E}_{q, p}^{\alpha}(A), \mathrm{X}\right) \leq c t^{\theta}|x|_{\left(\mathcal{E}_{q, p}^{\alpha}(A), \mathrm{X}\right)_{\theta, g}}, x \in\left(\mathcal{E}_{q, p}^{\alpha}(A), \mathrm{X}\right)_{\theta, g}
$$

Using (10), we have the inequality

$$
K\left(t, x ; \mathcal{E}_{q, p}^{\alpha}(A), \mathbf{X}\right) \leq c_{0} t^{\theta}|x|_{\mathcal{B}_{q, p, \tau}^{s, \alpha}(A)}^{\theta}, x \in \mathcal{B}_{q, p, \tau}^{s, \alpha}(A)
$$

with a constant $c_{0}(s, \tau)$.

Let $K_{\infty}\left(t, x ; \mathcal{E}_{q, p}^{\alpha}(A), \mathrm{X}\right)=\inf _{x=x^{0}+x^{1}} \max \left\{\left|x^{0}\right|_{\mathcal{E}_{q, p}^{\alpha}(A)}, t\left\|x^{1}\right\|\right\}$. The inequality $K_{\infty}\left(t, x ; \mathcal{E}_{q, p}^{\alpha}(A), \mathrm{X}\right) \leq K\left(t, x ; \mathcal{E}_{q, p}^{\alpha}(A), \mathrm{X}\right)$ yields

$$
t^{-\theta} K_{\infty}\left(t, x ; \mathcal{E}_{q, p}^{\alpha}(A), \mathrm{X}\right) \leq c_{0}|x|_{\mathcal{B}_{q, p, \tau}^{s, \alpha}(A)}^{\theta}, x \in \mathcal{B}_{q, p, \tau}^{s, \alpha}(A)
$$

By Lemma 7.1.2 [8] for every $t>0$ there exists $v>0$ such that

$$
K_{\infty}\left(t, x ; \mathcal{E}_{q, p}^{\alpha}(A), \mathbf{X}\right)=v, \lim _{\mu \downarrow v} E_{q, p}^{\alpha}(\mu, x)=E_{q, p}^{\alpha}(v+0, x) \leq v / t
$$

As a result, $v^{1-\theta}\left[E_{q, p}^{\alpha}(v, x)\right]^{\theta} \leq t^{-\theta} K_{\infty}\left(t, x ; \mathcal{E}_{q, p}^{\alpha}(A), \mathrm{X}\right)$. Using (13), we have

$$
v^{1-\theta}\left[E_{q, p}^{\alpha}(v, x)\right]^{\theta} \leq c_{0}|x|_{\mathcal{B}_{q, p, \tau}^{s, \alpha}(A)}^{\theta}
$$

If $s=(1-\theta) / \theta$ then $v^{s} E_{q, p}^{\alpha}(v, x) \leq c_{0}^{1 / \theta}|x|_{\mathcal{B}_{q, p, \tau}^{s, \alpha}(A)}$ for all $x \in \mathcal{B}_{q, p, \tau}^{s, \alpha}(A)$. Taking $c_{2}=c_{0}^{1 / \theta}$, we obtain the required inequality $(12)$.

Now we consider a spectral approximation problem for the positive operator with the point spectrum. We say that $A$ is the operator with the point spectrum if $\sigma(A)$ consists of isolated eigenvalues $\lambda_{j}(j \in \mathbb{N})$ with a unique boundary point at infinity. Moreover, every root subspace $\mathcal{R}_{\lambda_{j}}(A)$ corresponding to $\lambda_{j} \in \sigma(A)$ is finite dimensional.

For $m, n \in \mathbb{N}$ we denote by $\mathcal{R}_{m, n}^{\nu}(A)$ the complex linear span in $\mathrm{X}$ of all $\left\{\mathcal{R}_{\lambda_{j}}(A):\left|\lambda_{j}\right|<\min \left(\nu^{1 /(m+1)}, \nu^{1 /(n+1)}\right)\right\}$. Let $\mathcal{H}_{\lambda_{j}}(A)=\left\{x \in \mathrm{D}^{\infty}(A):\right.$ $\left.\left(\lambda_{j} I-A\right) x=0\right\}$ be a subspace of eigenvectors corresponding to $\lambda_{j} \in \sigma(A)$ and $\mathcal{H}_{m, n}^{\nu}(A)$ be a complex linear span of all $\mathcal{H}_{\lambda_{j}}(A)$ such that

$$
\left|\lambda_{j}\right|=\min \left(\nu^{1 /(m+1)}, \nu^{1 /(n+1)}\right)
$$

$\lambda_{j} \in \sigma(A)$. 
Theorem 5. Let $A$ be a positive operator with the point spectrum, $m, n \in$ $\mathbb{N}, \alpha=m(1-\theta)+n \theta$ and $0<\theta<1$. Then the following inequalities hold:

$$
\begin{gathered}
\inf \left\{\left\|x-x^{0}\right\|: x^{0} \in \mathcal{R}_{m, n}^{\nu}(A)\right\} \leq c \nu^{-s}|x|_{\mathcal{B}_{q, q, \tau}^{s, \alpha}(A)}, \\
\inf \left\{\left\|x-x^{0}\right\|: x^{0} \in\left(\mathcal{R}_{m, n}^{\nu}(A), \mathcal{Q}_{m, n}^{\nu}(A)\right)_{1-1 / q, p}\right\} \leq c \nu^{-s}|x|_{\mathcal{B}_{q, p, \tau}^{s, \alpha}(A)},
\end{gathered}
$$

where $\mathcal{Q}_{m, n}^{\nu}(A)=\mathcal{R}_{m, n}^{\nu}(A) \oplus \mathcal{H}_{m, n}^{\nu}(A)$ and $\|\cdot\|_{\mathcal{R}_{m, n}^{\nu}(A)}:=\|\cdot\|_{\mathcal{E}_{1}^{\nu, \alpha}(A)}$, $\|$. $\left\|_{\mathcal{Q}_{m, n}^{\nu}(A)}:=\right\| \cdot \|_{\mathcal{E}_{\infty}^{\nu, \alpha}(A)}$.

Proof. If $\left|x^{0}\right|_{\mathcal{E}_{q, p}^{\alpha}(A)}=r\left(x^{0}\right)+\left\|x^{0}\right\| \leq v$, then $r\left(x^{0}\right) \leq v-\left\|x^{0}\right\|$, where $r\left(x^{0}\right)=\inf \left\{\nu>0: x^{0} \in \mathcal{E}_{q, p}^{\nu, \alpha}(A)\right\}$. So, $x^{0} \in \mathcal{E}_{q, p}^{\nu, \alpha}(A)$ for all $\nu>0$ such that $r\left(x^{0}\right) \leq \nu \leq v-\left\|x^{0}\right\|$. Since $\mathcal{E}_{q, p}^{\nu, \alpha}(A) \subset \mathcal{E}_{q, p}^{v, \alpha}(A)$ that $x^{0} \in \mathcal{E}_{q, p}^{v, \alpha}(A)$. Hence, the inequality

$$
\inf \left\{\left\|x-x^{0}\right\|: x^{0} \in \mathcal{E}_{q, p}^{v, \alpha}(A)\right\} \leq E_{q, p}^{\alpha}(v, x), x \in \mathbf{X}, v>0
$$

holds. By Theorem 2.2 [9] for any $1 \leq q<\infty$ and $k \in \mathbb{Z}_{+}$we have

$$
\mathcal{E}_{q}^{\nu, k}(A)=\operatorname{span}\left\{\mathcal{R}_{\lambda_{j}}(A):\left|\lambda_{j}\right|^{k+1}<\nu\right\}
$$

Applying (17), (7) and Theorem 1.15.3 [7], we obtain

$$
\begin{aligned}
\mathcal{E}_{q}^{\nu, \alpha}(A) & =\mathcal{E}_{q, \theta}^{\nu,[m, n]}(A)=\left[\mathcal{E}_{q_{0}}^{\nu, m}(A), \mathcal{E}_{q_{1}}^{\nu, n}(A)\right]_{\theta} \\
& =\mathcal{E}_{q_{0}}^{\nu, m}(A) \cap \mathcal{E}_{q_{1}}^{\nu, n}(A)=\mathcal{R}_{m, n}^{\nu}(A)
\end{aligned}
$$

with $\alpha=m(1-\theta)+n \theta$. Using (18), (16) and (12), we obtain (14).

Now let us prove the equality

$$
\mathcal{E}_{\infty}^{\nu, \alpha}(A)=\mathcal{Q}_{m, n}^{\nu}(A)
$$

Using [10, Lemma 1], we have

$$
\mathcal{E}_{\infty}^{\nu, \alpha}(A) \subset \operatorname{span}\left\{\mathcal{R}_{\lambda_{j}}(A):\left|\lambda_{j}\right| \leq \min \left(\nu^{1 /(m+1)}, \nu^{1 /(n+1)}\right)\right\} .
$$

Then it is sufficient to prove the equality $\mathcal{H}_{\lambda_{j}}(A)=\mathcal{E}_{\infty}^{\nu, 0}(A) \cap \mathcal{R}_{\lambda_{j}}(A)$ for indices $j$ with $\left|\lambda_{j}\right|=\nu$. Assume that this equality is not true. Then there exist root vectors $x_{0}, \ldots, x_{r}$ corresponding to $\lambda_{j}$ such that $\left|\lambda_{j}\right|=\nu$ and $x_{r} \in \mathcal{E}_{\infty}^{\nu, 0}(A)$, $r \geq 1$. From the equality

$$
A^{k} x_{r}=\sum_{i=0}^{r}\left(\begin{array}{l}
k \\
i
\end{array}\right) \lambda_{j}^{k-i} x_{r-i}, \quad k \geq r
$$


it follows that

$$
\lim _{k \rightarrow \infty} \frac{\left\|A^{k} x_{r}\right\|}{\left(\begin{array}{l}
k \\
r
\end{array}\right) \nu^{k}}=\nu^{-r}\left\|x_{0}\right\| .
$$

Since $x_{0} \neq 0$ then $\nu^{-r}\left\|x_{0}\right\| \neq 0$ and $x_{r} \notin \mathcal{E}_{\infty}^{\nu, 0}(A)$. So, the equality $\mathcal{H}_{\lambda_{j}}(A)=$ $\mathcal{E}_{\infty}^{\nu, 0}(A) \cap \mathcal{R}_{\lambda_{j}}(A)$ for $j:\left|\lambda_{j}\right|=\nu$ holds. Thus, the equality (19) holds as well.

By (1), (18) and (19) we have

$$
\left(\mathcal{R}_{m, n}^{\nu}(A), \mathcal{Q}_{m, n}^{\nu}(A)\right)_{1-1 / q, p}=\mathcal{E}_{q, p}^{\nu, \alpha}(A)
$$

Using (20), (16) and (12), we obtain (15).

\section{References}

[1] M.L. Gorbachuk, V.I. Gorbachuk, Approximation of smooth vectors of a closed operator by entire vectors of exponential type, Ukrainian Math. J., 47 (1995), 713-726, doi: 10.1007/BF01059045.

[2] Ya.V. Radyno, Vectors of exponential type in operators calculus and differential equations, Differ. Equ., 21 (1985), 1559-1569.

[3] M.L. Gorbachuk, Ya.I. Grushka, S.M. Torba, Direct and inverse theorems in the theory of approximations by the Ritz method, Ukrainian Math. J., 57 (2005), 751-764, doi: 10.1007/s11253-005-0225-4.

[4] G.V. Radzievskii, Direct and converse theorems in problems of approximation by vectors of finite degree, Sb. Math. 189 (1998), 561-602, doi: 10.1070/SM1998v189n04ABEH000312.

[5] M. Dmytryshyn, O. Lopushansky, Bernstein-Jackson-type inequalities and Besov spaces associated with unbounded operators, J. Inequal. Appl., (2014), 2014:105, doi: 10.1186/1029-242X-2014-105.

[6] M. Dmytryshyn, Besov-Lorentz-type spaces and best approximations by exponential type vectors, Int. J. Math. Anal., 9 (2015), 779-786, doi: 10.12988/ijma.2015.5233.

[7] H. Triebel, Interpolation theory. Function spaces. Differential operators, North-Holland Publishing Company, Amsterdam-New York-Oxford, 1978.

[8] J. Bergh, J. Löfström, Interpolation Spaces, Springer-Verlag, Berlin-Heidelberg-New York, 1976.

[9] M. Dmytryshyn, O. Lopushansky, Operator calculus on the exponential type vectors of the operator with point spectrum, In: T. Banakh (ed.) General Topology in Banach Spaces, pp. 137-145. Nova Publ., New York (2001).

[10] G.V. Radzievskii, On the best approximations and rate of convergence of decompositions in the root vectors of an operator, Ukrainian Math. J., 49 (1997), 844-864, doi: $10.1007 / \mathrm{BF} 02513425$. 